\title{
EDITORIAL
}

\section{Editorial PIAM issue 02/2022}

\author{
Eujin Pei ${ }^{1}$
}

Accepted: 28 February 2022 / Published online: 3 March 2022

(c) The Author(s), under exclusive licence to Springer Nature Switzerland AG 2022

The Progress in Additive Manufacturing Journal 02/2022 is a 'bumper issue' that provides an unusually large number of 20 articles, once again covering a wide range of subjects within the scope of Additive Manufacturing. These papers include quality assessments of laser powder bed fusion builds from photodiode sensor measurements, improvement of green and sintered density of alumina parts fabricated by binder jetting, heat treatment of 17-4 PH stainless steel parts produced by binder jetting, mechanical properties of AlSi10Mg, use of coconut fibers in additive manufacturing, non-destructive evaluation and characterization of additively manufactured components, mechanical characterization of PBF-EB manufactured Ti6Al4V alloys, a review of recent trends of additive manufacturing, an overview of modeling techniques for metal powder bed fusion process chains, electrochemical deposition of metal printing, nanocomposites with added functionality in additive manufacturing, in situ alloying for powder bed fusion of metals, protocols for minimizing the leading edge bulge in stereolithography,
Schmitt-Kim additive manufacturing evaluation tree, generic roughness meta-model for material extrusion, flow rate ranges for spherical metallic powders, the influence of particle size distribution of monomodal $316 \mathrm{~L}$ powder on flowability and processability in powder bed fusion, additive manufacturing of NiTi shape memory parts, and lastly updates regarding standardization efforts of ISO/TC 261 "Additive Manufacturing" during the 17th plenary meeting.

Our Editorial Board members are working together to refresh the current scope of the journal. If you have suggestions, ideas or feedback, please write to me and get in touch. We would love to hear from readers and the scientific community.

Publisher's Note Springer Nature remains neutral with regard to jurisdictional claims in published maps and institutional affiliations.

Eujin Pei

eujin.pei@brunel.ac.uk

1 Brunel University London, Uxbridge, UK 